\title{
Outstanding Reviewer Award OR Spectrum 2019
}

๑) Springer-Verlag GmbH Germany, part of Springer Nature 2020

Zumbul Atan,

Eindhoven University

of Technology,

Eindhoven,

Netherlands

Rajan Batta,

University of Buffalo,

Buffalo, USA
Emrah Demir,

Cardiff University, Cardiff, United

Kingdom

\section{Emilia Gras,} Imperial College

London, London, United Kingdom
Sibel Salman,

Koc University,

Istanbul, Turkey

Alessio Trivella,

ETH Zurich,

Zurich,

Switzerland

Maria Besiou,

Kühne Logistics

University,

Hamburg, Germany

Publisher's Note Springer Nature remains neutral with regard to jurisdictional claims in published maps and institutional affiliations. 